\title{
A fully adaptive numerical approximation for a two-dimensional epidemic model with nonlinear cross-diffusion
}

\author{
Stefan Berres ${ }^{\mathrm{a}}$, Ricardo Ruiz-Baier ${ }^{\mathrm{b}}$ \\ ${ }^{a}$ Departamento de Ciencias Matemática y Física, Facultad de Ingeniería, Universidad Católica de Temuco, Temuco, Chile. \\ ${ }^{b}$ Modeling and Scientific Computing CMCS-MATHICSE-SB, École Polytechnique Fédérale de Lausanne, CH-1015 Lausanne, Switzerland.
}

\begin{abstract}
An epidemic model is formulated by a reaction-diffusion system where the spatial pattern formation is driven by crossdiffusion. Whereas the reaction terms describe the local dynamics of susceptible and infected species, the diffusion terms account for the spatial distribution dynamics. For both self-diffusion and cross-diffusion nonlinear constitutive assumptions are suggested. To simulate the pattern formation two finite volume formulations are proposed, which employ a conservative and a non-conservative discretization, respectively. An efficient simulation is obtained by a fully adaptive multiresolution strategy. Numerical examples illustrate the impact of the cross-diffusion on the pattern formation.
\end{abstract}

Key words: Epidemic model, reaction-diffusion equation, cross-diffusion, fully adaptive multiresolution $2000 \mathrm{MSC}: 35 \mathrm{~K} 55,92 \mathrm{D} 30,65 \mathrm{M} 08,65 \mathrm{M} 50$

\section{Introduction}

The knowledge of spreading dynamics of infectious diseases helps to design prevention measures [17, 34]. A generic model category for the quantitative description of the epidemic evolution dynamics by an ordinary differential equation are the so-called SIR models, which classify a population into 'susceptible' (S), 'infected' (I) and 'recovered' (R) subgroups and balance the changes between these. One very early and simple prototype of a SIR-model is due to Kermack-McKendrick [28]. It describes the population evolution by the system of ordinary differential equations

$$
\frac{d S}{d t}=-\alpha S I, \quad \frac{d I}{d t}=\alpha S I-\beta I, \quad \frac{d R}{d t}=\beta I
$$

where $\alpha>0$ is the infection rate and $\beta>0$ the recovery rate. There are several suggestions for improving the specification of these ODE-dynamics (see e.g. [29, 32, 47]), and structural modifications like SIR-models in networks [33] or with delays [26]. See also [14, 27, 39] for a more general reflection on mathematical modeling in epidemiology. A key issue in epidemic modeling is the formation of spatial patterns [23]. Based on a general setting for the two-dimensional reaction-diffusion framework for epidemic processes [48], there are several suggestions for the combination of the system of ordinary differential equations of the SIR-model with a spatially two-dimensional diffusion equations of the involved variables [25, 31, 36, 40, 45]. Moreover, several contributions have been proposed to study pattern formation induced by cross-diffusion [6, 38, 46]. In addition to a fundamental existence proof for general reaction-diffusion systems [15], there are several approaches to analyze reaction-diffusion equations with one single "cross-diffusion" that lead to a system with upper triangular diffusion matrix [2, 3, 16, 44]; the latter extends the method of [35] for a diagonal diffusion matrix. The structure of an upper triangular diffusion matrix has also been utilized in the existence analysis for systems of convection-diffusion equation with both Dirichlet and Neumann boundary conditions (see e.g. [8, 19, 20]). Besides numerous contributions to the development of numerical methods to solve reaction-diffusion equations in related contexts [42, 49], convergence proofs of associated finite volume schemes [1,7] and finite element formulations [4, 21] have been provided.

Email addresses: sberres@uct.cl (Stefan Berres), ricardo.ruiz@epfl.ch (Ricardo Ruiz-Baier) 
The goal of this contribution is, on the one hand, to generate pattern formation in an epidemic model by a crossdiffusion term, and, on the other hand to prevent blow-up by the a non-linear limitation of the cross-diffusion. These assumptions are designed to qualitatively reflect psychological behavior. The cross-diffusion term has the interpretation that the susceptible population moves away from increasing gradients of the infected population. In addition, it is assumed that the cross-diffusion effect depends on the local population density. For the nonlinear cross-diffusion it is assumed that there exists carefreeness at a small and fatalism at a high total population number. At carefreeness and fatalism the susceptible population decreases its tendency to avoid agents of the infected population. Such an avoidance is most effective for intermediate (neither too small nor too large) population numbers.

The novelty of this article consists in the design of nonlinear self-diffusion and cross-diffusion functions, the appropriate discretization of these functions and the usage of adaptive strategies for this particular class of reaction-diffusion equations. Thereby, a robust and efficient simulation tool allowing any kind of nonlinear constitutive assumptions is promoted. The discretization of linear terms leaves an ambiguity on their generalization to the appropriate discretization of nonlinear terms. In particular there is the choice with respect to conservative versus non-conservative discretization. For both cases a discretization is suggested and tested.

The new nonlinear feature of the equation system is mainly embodied in the cross-diffusion coefficient, which generates pattern formation. The reaction part of the reaction-diffusion equation, which can be formulated as an ordinary differential equation, is asymptotically stable, i.e. converges to a stationary state. Without a cross-diffusion term the whole reaction-diffusion equation would in the same way converge to the stationary state. The cross-diffusion term counteracts this stability, leading to pattern formation. The proposed simulation tool allows to qualitatively study the effect of non-linear cross-diffusions.

In the subsequent Sections, the precise equations of the reaction-diffusion system are stated and nonlinear self- and cross-diffusion terms are proposed (Section 2). Given that pattern formation leads to a solution structure that shows sharp interfaces, a fully adaptive multiresolution scheme is employed (Section 3) on the basis of both a conservative and a non-conservative discretization. Finally, the effects of different parameter and model choices are compared and discussed (Section 4).

\section{Epidemic model with cross diffusion}

The two-dimensional reaction-diffusion system describing spatial epidemic dynamics with cross-diffusion is written as

$$
\begin{aligned}
& u_{t}=f(u, v)+\nabla \cdot(a(u) \nabla u)+\nabla \cdot(c(u, v) \nabla v), \\
& v_{t}=g(u, v)+\nabla \cdot(b(v) \nabla v), \quad \text { in } \quad \Omega_{T}=\Omega \times(0, T),
\end{aligned}
$$

where $u$ and $v$ denote the populations of susceptible and infected persons, respectively. No external input is imposed, therefore on the physical domain boundary $\partial \Omega$ it holds the Neumann boundary condition

$$
(a(u) \nabla u+c(u, v) \nabla v) \cdot n=0, \quad(b(v) \nabla u) \cdot n=0,
$$

where $n$ is the outer normal vector to the physical domain boundary. In the system (2.1), an additional equation for the recuperated population is omitted because the model does not consider their feedback on the susceptible or infected population. With the notation

$$
\boldsymbol{u}=\left(\begin{array}{l}
u \\
v
\end{array}\right), \quad \boldsymbol{f}(\boldsymbol{u})=\left(\begin{array}{l}
f(u, v) \\
g(u, v)
\end{array}\right), \quad \boldsymbol{a}(\boldsymbol{u})=\left(\begin{array}{cc}
a(u) & c(u, v) \\
0 & b(v)
\end{array}\right)
$$

the system 2.1] can be written in compact form as

$$
\frac{d}{d t} \boldsymbol{u}=\boldsymbol{f}(\boldsymbol{u})+\nabla \cdot(\boldsymbol{a}(\boldsymbol{u}) \nabla \boldsymbol{u})
$$

The model enforces phase separation since the susceptible species avoid the infected by a cross-diffusion term $\nabla \cdot(c(u, v) \nabla v)$ [45]. The cross-diffusion term directs the flow in the opposite direction of the gradient $\nabla v$. Whenever there is an increase of the amount of infected then the susceptible agents move away from the direction of the increasing gradient. 


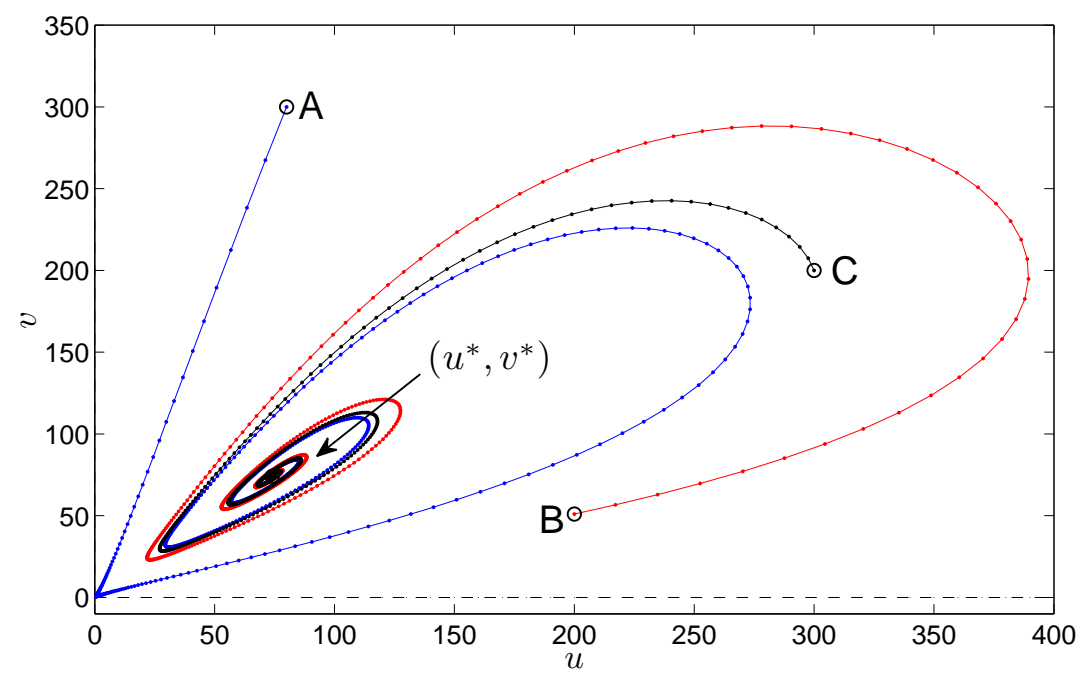

Figure 1: Phase portrait for the ODE system associated to 2.1, 2.2. Three trajectories are displayed starting from the states $A=(80,300)$, $B=(200,50)$ and $C=(300,200)$ and reaching the equilibrium point $\left(u^{*}, v^{*}\right)$. The parameters correspond to those used in Example 1A.

The reaction terms are considered to be given by the following specifications (see e.g. [45])

$$
f(u, v)=r u(1-u / K)-\beta \frac{u v}{u+v}, \quad g(u, v)=\beta \frac{u v}{u+v}-k v,
$$

where the model parameters are the carrying capacity of the susceptible species $K, r$ is the intrinsic birth rate, $\beta$ is the rate of disease transmission, and $k$ represents the recovery rate of the infected species.

The equilibrium points are pairs $(u, v)$ such that $f(u, v)=0$ and $g(u, v)=0$. For $(2.2)$, the equilibrium points are $(0,0)$ (trivial equilibrium), $(K, 0)$, which corresponds to the disease-free point, and $\left(u^{*}, v^{*}\right)$, which corresponds to an endemic stationary state that is explicitly given by

$$
\left(u^{*}, v^{*}\right)=\left(\frac{K(r-\beta+k)}{r}, \frac{K(r-\beta+k)(\beta-k)}{r k}\right) .
$$

In Figure 1, the phase portrait of the ODE system associated to 2.1), 2.2), i.e. the system (2.1) with reaction part but without diffusion part, is shown with several choices of initial data. The system is asymptotically stable in the sense that if the initial data are chosen close to the non-trivial equilibrium then the solution converges to this equilibrium. This can be shown analytically by demonstrating that the Jacobian matrix

$$
F^{*}=F\left(u^{*}, v^{*}\right)=\left(\begin{array}{ll}
\frac{\partial f}{\partial u}\left(u^{*}, v^{*}\right) & \frac{\partial f}{\partial v}\left(u^{*}, v^{*}\right) \\
\frac{\partial g}{\partial u}\left(u^{*}, v^{*}\right) & \frac{\partial g}{\partial v}\left(u^{*}, v^{*}\right)
\end{array}\right)
$$

has eigenvalues $\lambda_{1}, \lambda_{2}$ with negative real part, which can be checked by

$$
\operatorname{tr}(F)=\frac{\partial f}{\partial u}+\frac{\partial g}{\partial v}<0, \quad|F|=\frac{\partial f}{\partial u} \frac{\partial g}{\partial v}-\frac{\partial f}{\partial v} \frac{\partial g}{\partial u}>0 .
$$

The Jacobian matrix corresponding to the specification 2.2 is

$$
F(u, v)=\left(\begin{array}{cc}
r\left(1-2 \frac{u}{K}\right)-\frac{\beta v^{2}}{(u+v)^{2}} & -\frac{\beta u^{2}}{(u+v)^{2}} \\
\frac{\beta v^{2}}{(u+v)^{2}} & \frac{\beta u^{2}}{(u+v)^{2}}-k
\end{array}\right) .
$$

From now on, as model variants we will consider diffusion terms which are linear (Model 1) and nonlinear (Model 2). For better comparison, in Model 2 the same reaction kinetics as for Model 1 are used. 


\subsection{Model 1}

For Model 1 we assume linear diffusion functions, where the diffusion coefficients are chosen to be the constants

$$
a(u)=a_{0}, \quad b(v)=b_{0}, \quad c(u, v)=c_{0},
$$

which adopts the setting of [45].

\subsection{Model 2}

In Model 2 we propose nonlinear model variants of the parametric functions. The self-diffusion terms are chosen to have the form

$$
a(u)=a_{0} u^{m}, \quad b(v)=b_{0} v^{m} .
$$

By the assumption that $m \in(-1,0)$ a degressive growth is described since

$$
\partial_{u} a(u)<0, \quad \partial_{v} b(v)<0 \text { for all } u, v .
$$

The biological interpretation is that the tendency to avoid crowds reduces with higher numbers as the population "gets used" to them. Using the notation of the Laplace operator,

$$
\Delta A(u)=\nabla \cdot(a(u) \nabla u), \quad \Delta B(v)=\nabla \cdot(b(v) \nabla v),
$$

we have

$$
A(u)=A_{0} u^{n}=\frac{a_{0}}{m+1} u^{m+1}, \quad B(v)=B_{0} v^{n}=\frac{b_{0}}{m+1} v^{m+1} .
$$

where $A(u)$ and $B(v)$ are sub-linear functions. For $m=0$ the functions 2.5) of Model 2 boil down to those of the linear Model 1 (2.4) as

$$
A(u)=a_{0} u, \quad B(v)=b_{0} v .
$$

The construction of a conservative or non-conservative discretization depends roughly speaking on whether the differences are based on the formulation of the left-hand or right-hand side of [2.6, , respectively.

A nonlinear cross-diffusion function implements a situation-dependent tendency of the susceptible population to avoid the infected population. This situation-dependent behavior reflects an average psychological disposition. An approach to model such a disposition is as follows. From the perspective of the susceptible population, avoidance is pursued whenever there is a detectable fraction of the infected population. For a small number of populations the necessary awareness has not been matured or is temporarily not active, since then there is no vital urgency for self-protection. In the other extreme, at large population numbers, such a selective detection is neither possible nor makes sense, since there is less, or even no chance to avoid infection in the crowd. The population number affects the conscious disposition of avoidance. Therefore, the cross-diffusion coefficient is designed to be negligible for both small and large number of populations, which is imposed by the following constraints

$$
\begin{aligned}
& c(u, 0)=0, \quad c(0, v)=0, \quad \text { for all } \quad u, v \in \mathbb{R}, \\
& c(u, v)=0 \quad \text { for } \quad v \geq V(u),
\end{aligned}
$$

where $V$ is a Lipschitz continuous monotonically decreasing function with a zero for $u>0$. For example, one might choose $V(u)=c_{1}-u$ with $c_{1}>0$. By these constraints, the reaction-diffusion equation with cross-diffusion 2.1 degenerates into an equation without cross-diffusion outside the domain

$$
\tilde{\Omega}:=\{(u, v): u, v \geq 0, v<V(u)\} .
$$

The constraints (2.7) are satisfied, for example, by the cuadratic function

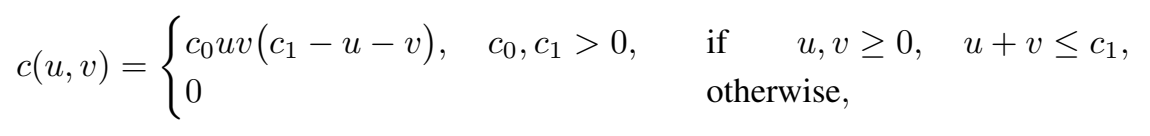


which is a convex function that takes its global maximum in

$$
(\hat{u}, \hat{v})=\left(\frac{c_{1}}{3}, \frac{c_{1}}{3}\right) .
$$

In the sequel some supporting arguments for the constraints (2.7) and in particular for the nonlinear model (2.8) are summarized. First of all, the constraint $c(u, 0)=0$ for all $u$ corresponds to carefreeness; during the absence, and also in the case of a small number of infected persons, the consciousness of the danger of the disease is not sufficiently present, even though there might be are some single dangerous intercoursesf. The constraint $c(0, v)=0$ for all $u$ is not only set for symmetry reasons, since, at a small number of susceptible agents, they have little chance to form a groups consciousness on the importance of a separation from the infected population; instead, the susceptible population is absorbed by the infected population. The maximum $(\hat{u}, \hat{v})$ has the interpretation that there is most avoidance when there is a fairly equal mixing of susceptible and infected population, whereas at small population numbers there is no need and at large total population numbers no possibility for avoidance.

In the situation of a large concentration of persons there is little possibility of a selective avoidance. Since the infected species is present anywhere and thus cannot be sustainably avoided in the crowd, there is small to no possibility to keep distance from the infected species. Thus, above a certain treshold 'upper' population number fatalism rules. This fatalism is modelled by the assumption that the cross-diffusion coefficient vanishes then above a treshold population number. This upper population bound is set by the function $V$ such that $c_{1}$ sets a maximum population, where, in the case that $c_{1}=u+v$, total fatalism rules.

A formal property of the constraints (2.7) is the that the cross-diffusion is switched off at a certain finite total population. By this setting, it is prevented that the population attains (unrealistic) local population peaks. In fact, by this limitation of the cross-diffusion, a maximum principle for the system 2.1] is imposed. The switch-off of the diffusion term can be seen in the context of equations with strongly degenerate diffusion, where the diffusion function is set to zero on an interval) as proposed in [5, 10]. There, sharper interfaces have been evidenced by the degenerate diffusion.

\section{Numerical approximation}

In order to provide a space-adaptive numerical scheme, we apply the technique of fully adaptive multiresolution [5, 11] constructed on the basis of a reference finite volume approximation [18] (see also e.g. [37] for a survey on multiresolution methods for PDEs). The success of this approach mainly relies on the strategy used for storing only the relevant information. The numerical approximation obtained in each time step is represented (and also computed) using a dynamically evolving adaptive mesh which is generated from a sequence of nested grids. An appropriate smoothness analysis of the solution is performed using a wavelet decomposition and such information on the local smoothness is used to locally adapt the mesh and the numerical scheme. Essentially, positions related to small wavelet coefficients may be discarded, allowing for substantial data and CPU-time compression.

\section{Admissible rectangular meshes}

These ideas are made precise, first by introducing a nested hierarchy of grids $\mathcal{T}^{0} \subset \cdots \subset \mathcal{T}^{H}$, where each grid $\mathcal{T}^{\ell}, \ell=0, \ldots, H$ is assumed to be an admissible rectangular mesh. The index $\ell=0$ corresponds to the coarsest and $\ell=H$ to the finest resolution level, which is fixed and chosen large enough at the beginning of the algorithm. That is, a partition of $\Omega$ formed by control volumes $K^{\ell}$ (open rectangles of maximum diameter $h_{K^{\ell}}$ ), constrained by the condition that the segment joining the centers of two neighboring control volumes $x_{K^{\ell}}$ and $x_{L^{\ell}}$ must be orthogonal to the corresponding interface $\sigma=\sigma\left(K^{\ell}, L^{\ell}\right)$. The interface length is denoted by $|\sigma|=\left|\sigma\left(K^{\ell}, L^{\ell}\right)\right|$.

For instance, the mesh on the right of Figure 2 is admissible, and meshes as the one on the left are ruled out by the orthogonality condition. By $\mathcal{E}\left(K^{\ell}\right)$ we denote the set of edges of $K^{\ell}, \mathcal{E}_{\text {int }}\left(K^{\ell}\right)$ corresponds to those is in the interior of $\mathcal{T}^{\ell}$ and $\mathcal{E}_{\text {ext }}\left(K^{\ell}\right)$ is the set of edges of $K^{\ell}$ lying on the boundary $\partial \Omega$, i.e.,

$$
\mathcal{E}(K)=\mathcal{E}_{\text {int }}\left(K^{\ell}\right) \cup \mathcal{E}_{\text {ext }}\left(K^{\ell}\right), \quad \mathcal{E}_{\text {int }}\left(K^{\ell}\right) \cap \mathcal{E}_{\text {ext }}\left(K^{\ell}\right)=\varnothing \quad \text { for all } K^{\ell} \in \mathcal{T}^{\ell} .
$$

By $\mathcal{E}_{\text {int }}^{\ell}$ and $\mathcal{E}_{\text {ext }}^{\ell}$ we will denote the sets of all edges in the interior of $\mathcal{T}^{\ell}$ and lying on the boundary $\partial \Omega$, respectively. For a given finite volume $K^{\ell}$, we denote by $N\left(K^{\ell}\right)$ the set of neighbors of $K^{\ell}$ which share a common edge with $K^{\ell}$. For all $L^{\ell} \in N\left(K^{\ell}\right), d\left(K^{\ell}, L^{\ell}\right)$ denotes the distance between $x_{K^{\ell}}$ and $x_{L^{\ell}}$. 

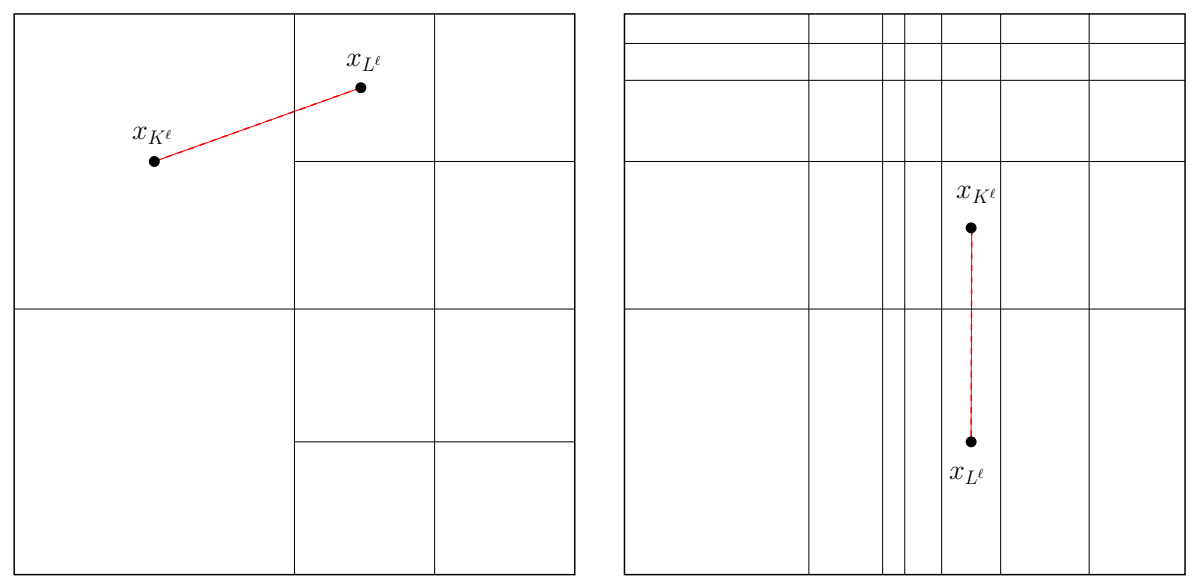

Figure 2: Sketch of non-admissible (left) and admissible rectangular meshes (right).

\section{Two one-level finite volume methods}

In order to define the discrete marching formula for (2.1), we choose an admissible discretization of $\Omega_{T}$ consisting of an admissible mesh $\mathcal{T}^{\ell}$ of $\Omega$ and a time step size $\Delta t>0$. We may choose $N>0$ as the smallest integer such that $N \Delta t \geq T$, and set $t^{n}:=n \Delta t$ for $n \in\{0, \ldots, N\}$.

We denote the cell averages of $u$ and $v$ on $K^{\ell} \in \mathcal{T}^{\ell}$ at time $t=t^{n}$ by the respective expressions

$$
u_{K^{\ell}}^{n}:=\frac{1}{\left|K^{\ell}\right|} \int_{K^{\ell}} u\left(x, t^{n}\right) d x, \quad v_{K^{\ell}}^{n}:=\frac{1}{\left|K^{\ell}\right|} \int_{K^{\ell}} v\left(x, t^{n}\right) d x .
$$

Furthermore, we define the coefficients

$$
f_{K^{\ell}}^{n}:=f\left(u_{K^{\ell}}^{n}, v_{K^{\ell}}^{n}\right), g_{K^{\ell}}^{n}:=g\left(u_{K^{\ell}}^{n}, v_{K^{\ell}}^{n}\right), a_{K^{\ell}}^{n}:=a\left(u_{K^{\ell}}^{n}\right), b_{K^{\ell}}^{n}:=b\left(v_{K^{\ell}}^{n}\right), c_{K^{\ell}}^{n}:=c\left(u_{K^{\ell}}^{n}, v_{K^{\ell}}^{n}\right),
$$

For constant coefficient functions 2.4 one has $a_{K^{\ell}}^{n}=a_{0}, b_{K^{\ell}}^{n}=b_{0}, c_{K^{\ell}}^{n}=c_{0}$ on all cells $K^{\ell}$ and time steps $n$. The computation starts from the initial cell averages

$$
u_{K^{\ell}}^{0}=\frac{1}{\left|K^{\ell}\right|} \int_{K^{\ell}} u_{0}(x) d x, \quad v_{K^{\ell}}^{0}=\frac{1}{\left|K^{\ell}\right|} \int_{K^{\ell}} v_{0}(x) d x .
$$

The resulting finite volume scheme for the approximation of (2.1), defined on the multiresolution level $l$ assumes values $u_{K^{\ell}}^{n}$ and $v_{K^{\ell}}^{n}$ for all $K^{\ell} \in \mathcal{T}^{\ell}$ at time $t=t^{n}$ and determines $u_{K^{\ell}}^{n+1}$ and $v_{K^{\ell}}^{n+1}$ for all $K^{\ell} \in \mathcal{T}^{\ell}$ at time $t=t^{n+1}=t^{n}+\Delta t$ by a marching formula. For linear coefficients (2.4) the system (2.1) is discretized as

$$
\begin{aligned}
& \left|K^{\ell}\right| \frac{u_{K^{\ell}}^{n+1}-u_{K^{\ell}}^{n}}{\Delta t}=\left|K^{\ell}\right| f_{K^{\ell}}^{n}+\sum_{\sigma \in \mathcal{E}_{\text {int }}\left(K^{\ell}\right)} \frac{\left|\sigma\left(K^{\ell}, L^{\ell}\right)\right|}{d\left(K^{\ell}, L^{\ell}\right)}\left\{a_{0}\left(u_{L^{\ell}}^{n}-u_{K^{\ell}}^{n}\right)+c_{0}\left(v_{L^{\ell}}^{n}-v_{K^{\ell}}^{n}\right)\right\} \\
& \left|K^{\ell}\right| \frac{v_{K^{\ell}}^{n+1}-v_{K^{\ell}}^{n}}{\Delta t}=\left|K^{\ell}\right| g_{K^{\ell}}^{n}+\sum_{\sigma \in \mathcal{E}_{\text {int }}\left(K^{\ell}\right)} \frac{\left|\sigma\left(K^{\ell}, L^{\ell}\right)\right|}{d\left(K^{\ell}, L^{\ell}\right)} b_{0}\left(u_{L^{\ell}}^{n}-u_{K^{\ell}}^{n}\right) .
\end{aligned}
$$

This marching formula is valid for all cells and in particular for the boundary cells. The no-slip boundary condition is considered automatically by not considering boundary fluxes, such that they are automatically set to zero.

For nonlinear coefficient functions the generalization of $(3.1)$ is not uniquely determined. Therefore, two versions are suggested, which are denoted by scheme A and scheme B, respectively. Scheme A has the form 


$$
\begin{aligned}
& \left|K^{\ell}\right| \frac{u_{K^{\ell}}^{n+1}-u_{K^{\ell}}^{n}}{\Delta t}=\left|K^{\ell}\right| f_{K^{\ell}}^{n}+\sum_{\sigma \in \mathcal{E}_{\text {int }}\left(K^{\ell}\right)} \frac{\left|\sigma\left(K^{\ell}, L^{\ell}\right)\right|}{d\left(K^{\ell}, L^{\ell}\right)}\left\{\frac{\left(a_{L^{\ell}}^{n}+a_{K^{\ell}}^{n}\right)}{2}\left(u_{L^{\ell}}^{n}-u_{K^{\ell}}^{n}\right)+\frac{2 c_{K^{\ell}}^{n} c_{L^{\ell}}^{n}}{c_{K^{\ell}}^{n}+c_{L^{\ell}}^{n}}\left(v_{L^{\ell}}^{n}-v_{K^{\ell}}^{n}\right)\right\} \\
& \left|K^{\ell}\right| \frac{v_{K^{\ell}}^{n+1}-v_{K^{\ell}}^{n}}{\Delta t}=\left|K^{\ell}\right| g_{K^{\ell}}^{n}+\sum_{\sigma \in \mathcal{E}_{\text {int }}\left(K^{\ell}\right)} \frac{\left|\sigma\left(K^{\ell}, L^{\ell}\right)\right|}{d\left(K^{\ell}, L^{\ell}\right)} \frac{\left(b_{L^{\ell}}^{n}+b_{K^{\ell}}^{n}\right)}{2}\left(v_{L^{\ell}}^{n}-v_{K^{\ell}}^{n}\right) .
\end{aligned}
$$

Whereas the coefficient functions $a(u, v)$ and $b(u, v)$ are averaged, for the coefficient function $c(u, v)$ the exchange coefficient in the cross-diffusion term is computed by the following harmonic mean formula (see e.g. [18])

$$
c\left(\left(u_{K^{\ell}}^{n}, v_{K^{\ell}}^{n}\right) ;\left(u_{L^{\ell}}^{n}, v_{L^{\ell}}^{n}\right)\right):=\frac{2 c_{K^{\ell}}^{n} c_{L^{\ell}}^{n}}{c_{K^{\ell}}^{n}+c_{L^{\ell}}^{n}} .
$$

We note that (3.3) is consistent in the sense that $c((u, v) ;(u, v))=c(u, v)$.

Even though scheme A looks reasonable, it is not conservative. For the discretization of conservation laws, it is well known that a non-conservative discretization might converge to a wrong solution (see e.g. [24, 30]). For nonconservative equations a possible remedy is the formulation of path-conservative schemes [13, 41]. For parabolic equations (as treated here) there is a similar situation, which demands a careful consideration. In [9, Figure 2] it is demonstrated that a non-conservative discretization of the parabolic term can produce spurious solutions; our scheme $\mathrm{A}$ is a two-dimensional version of the non-conservative discretization specified in their formula (12). Therefore, we try to mimic in scheme B the conservative discretization, see e.g. their formula (13). In other words, scheme B avoids averaged transmission coefficients, instead finite differences are calculated in terms of the antiderivatives $A, B, C$,

$$
\frac{\partial A(u)}{\partial u}=a(u), \quad \frac{\partial B(v)}{\partial v}=b(v), \quad \frac{\partial C(u, v)}{\partial v}=c(u, v),
$$

that retain the local nonlinear properties. Differencing with respect to these antiderivatives gives to the resulting scheme a conservative form. Scheme B has the form

$$
\begin{aligned}
& \left|K^{\ell}\right| \frac{u_{K^{\ell}}^{n+1}-u_{K^{\ell}}^{n}}{\Delta t}=\left|K^{\ell}\right| f_{K^{\ell}}^{n}+\sum_{\sigma \in \mathcal{E}_{\text {int }}\left(K^{\ell}\right)} \frac{\left|\sigma\left(K^{\ell}, L^{\ell}\right)\right|}{d\left(K^{\ell}, L^{\ell}\right)}\left\{\left(A_{L^{\ell}}^{n}-A_{K^{\ell}}^{n}\right)+\left(C_{L^{\ell}}^{n}-C_{K^{\ell}}^{n}\right)\right\}, \\
& \left|K^{\ell}\right| \frac{v_{K^{\ell}}^{n+1}-v_{K^{\ell}}^{n}}{\Delta t}=\left|K^{\ell}\right| g_{K^{\ell}}^{n}+\sum_{\sigma \in \mathcal{E}_{\text {int }}\left(K^{\ell}\right)} \frac{\left|\sigma\left(K^{\ell}, L^{\ell}\right)\right|}{d\left(K^{\ell}, L^{\ell}\right)}\left(B_{L^{\ell}}^{n}-B_{K^{\ell}}^{n}\right),
\end{aligned}
$$

with

$$
A_{K^{\ell}}^{n}:=A\left(u_{K^{\ell}}^{n}\right), \quad A_{L^{\ell}}^{n}:=A\left(u_{L^{\ell}}^{n}\right), \quad B_{K^{\ell}}^{n}:=B\left(u_{K^{\ell}}^{n}\right), \quad B_{L^{\ell}}^{n}:=B\left(u_{L^{\ell}}^{n}\right),
$$

which is justified due to the equalities

$$
\nabla A(u)=a(u) \nabla u, \quad \nabla B(v)=b(v) \nabla v .
$$

With respect to the definition of the coefficient $C$, there is the difficulty that

$$
\nabla C=\frac{\partial C}{\partial u} \nabla u+\frac{\partial C}{\partial v} \nabla v
$$

i.e. there remains the unresolvable term $\frac{\partial C}{\partial u} \nabla u$. Therefore a semi-averaged form of $C_{K^{\ell}}^{n}$ is built as

$$
C_{K^{\ell}}^{n}:=C\left(\bar{u}, v_{K^{\ell}}^{n}\right), \quad C_{L^{\ell}}^{n}:=C\left(\bar{u}, v_{L^{\ell}}^{n}\right), \quad \bar{u}=\bar{u}_{L^{\ell} K^{\ell}}=\frac{u_{L^{\ell}}^{n}+u_{K^{\ell}}^{n}}{2} .
$$


The antiderivative of $c(u, v)$ as defined in $(2.8)$ with respect to the variable $v$ is calculated as

$$
C(u, v)=c_{0} u \tilde{v}^{2}\left[\left(c_{1}-u\right) / 2-\tilde{v} / 3\right], \quad \tilde{v}=\min \left(v, c_{1}-u\right) .
$$

Scheme B should be more accurate than scheme A since the nonlinear functions are better approximated; more information is retained when differencing instead of simply calculating averages. For linear coefficient functions, when

$$
A_{K^{\ell}}^{n}:=a_{0} u_{K^{\ell}}^{n}, \quad B_{K^{\ell}}^{n}:=b_{0} u_{K^{\ell}}^{n}, \quad C_{K^{\ell}}^{n}:=c_{0} u_{K^{\ell}}^{n},
$$

both schemes (3.2) and (3.4) are the same and reduce to 3.1). The schemes 3.1, 3.2, (3.4) are explicit taking the form

$$
\left|K^{\ell}\right| \frac{u_{K^{\ell}}^{n+1}-u_{K^{\ell}}^{n}}{\Delta t}=F_{K^{\ell}}^{n}, \quad\left|K^{\ell}\right| \frac{v_{K^{\ell}}^{n+1}-v_{K^{\ell}}^{n}}{\Delta t}=G_{K^{\ell}}^{n},
$$

where the symbols $F_{K^{\ell}}^{n}$ and $G_{K^{\ell}}^{n}$ stand for the terms on the right-hand side in time step $n$. Denoting by $F_{K^{\ell}}^{n+1}$ and $G_{K^{\ell}}^{n+1}$ the corresponding expressions with unknowns evaluated in time step $n+1$, as more general time advancing strategy, we now formulate the well known $\theta$-method

$$
\left|K^{\ell}\right| \frac{u_{K^{\ell}}^{n+1}-u_{K^{\ell}}^{n}}{\Delta t}=(1-\theta) F_{K^{\ell}}^{n}+\theta F_{K^{\ell}}^{n+1}, \quad\left|K^{\ell}\right| \frac{v_{K^{\ell}}^{n+1}-v_{K^{\ell}}^{n}}{\Delta t}=(1-\theta) G_{K^{\ell}}^{n}+\theta F_{K^{\ell}}^{n+1},
$$

which allows us to arbitrarily choose an implicit or explicit marching formula. The constant $\theta, 0 \leq \theta \leq 1$ is the weight factor representing the degree of implicitness of the scheme. The values $\theta=0,1 / 2$ and 1 correspond to explicit, Crank-Nicolson, and implicit method respectively. The boundary scheme is specified accordingly, by using zero-flux boundary conditions.

Scheme A can be rewritten in the following way: For a given function $w$ and a given cell $K^{\ell}$, the discrete gradients $\nabla_{h} w$ on all edges $\sigma \in \mathcal{E}\left(K^{\ell}\right)$ are approximated by

$$
\begin{cases}\tau_{\sigma}^{n}\left(w_{L^{\ell}}^{n}-w_{K^{\ell}}^{n}\right) & \text { for } \sigma\left(K^{\ell}, L^{\ell}\right) \in \mathcal{E}_{\text {int }}\left(K^{\ell}\right), \\ 0 & \text { for } \sigma \in \mathcal{E}_{\text {ext }}\left(K^{\ell}\right),\end{cases}
$$

where the transmission coefficients $\tau_{\sigma}^{n}$ are defined by

$$
\tau_{a, \sigma}^{n}:=\frac{|\sigma|\left(a_{L^{\ell}}^{n}+a_{K^{\ell}}^{n}\right)}{2 d\left(K^{\ell}, L^{\ell}\right)}, \tau_{b, \sigma}^{n}:=\frac{|\sigma|\left(b_{L^{\ell}}^{n}+b_{K^{\ell}}^{n}\right)}{2 d\left(K^{\ell}, L^{\ell}\right)}, \tau_{c, \sigma}^{n}:=\frac{|\sigma| 2 c_{K^{\ell}}^{n} c_{L^{\ell}}^{n}}{d\left(K^{\ell}, L^{\ell}\right)\left[c_{K^{\ell}}^{n}+c_{L^{\ell}}^{n}\right]},
$$

for $\sigma=\sigma\left(K^{\ell}, L^{\ell}\right) \in \mathcal{E}_{\text {int }}\left(K^{\ell}\right)$. The $\theta$-method for scheme A thus becomes

$$
\begin{aligned}
\left|K^{\ell}\right| \frac{u_{K^{\ell}}^{n+1}-u_{K^{\ell}}^{n}}{\Delta t}=( & -\theta)\left[\left|K^{\ell}\right| f_{K^{\ell}}^{n}+\sum_{\sigma \in \mathcal{E}_{\text {int }}\left(K^{\ell}\right)} \tau_{a, \sigma}^{n}\left(u_{L^{\ell}}^{n}-u_{K^{\ell}}^{n}\right)+\sum_{\sigma \in \mathcal{E}_{\text {int }}\left(K^{\ell}\right)} \tau_{c, \sigma}^{n}\left(v_{L^{\ell}}^{n}-v_{K^{\ell}}^{n}\right)\right] \\
& +\theta\left[\left|K^{\ell}\right| f_{K^{\ell}}^{n+1}+\sum_{\sigma \in \mathcal{E}_{\text {int }}\left(K^{\ell}\right)} \tau_{a, \sigma}^{n}\left(u_{L^{\ell}}^{n+1}-u_{K^{\ell}}^{n+1}\right)+\sum_{\sigma \in \mathcal{E}_{\text {int }}\left(K^{\ell}\right)} \tau_{c, \sigma}^{n}\left(v_{L^{\ell}}^{n+1}-v_{K^{\ell}}^{n+1}\right)\right], \\
\left|K^{\ell}\right| \frac{v_{K^{\ell}}^{n+1}-v_{K^{\ell}}^{n}}{\Delta t}=( & 1-\theta)\left[\left|K^{\ell}\right| g_{K^{\ell}}^{n}+\sum_{\sigma \in \mathcal{\mathcal { E } _ { \text { int } }}\left(K^{\ell}\right)} \tau_{b, \sigma}^{n}\left(v_{L^{\ell}}^{n}-v_{K^{\ell}}^{n}\right)\right] \\
& +\theta\left[\left|K^{\ell}\right| g_{K^{\ell}}^{n+1}+\sum_{\sigma \in \mathcal{\mathcal { E }}_{\text {int }}\left(K^{\ell}\right)} \tau_{b, \sigma}^{n}\left(v_{L^{\ell}}^{n+1}-v_{K^{\ell}}^{n+1}\right)\right],
\end{aligned}
$$




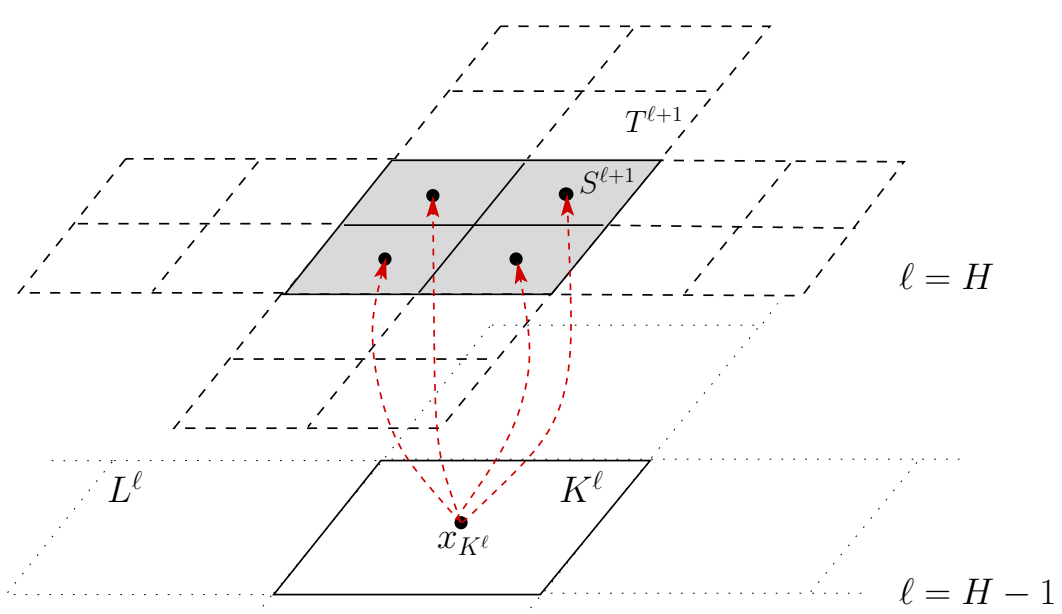

Figure 3: Sketch of a graded tree structure. Here $K^{\ell}$ is a parent node on level $\ell=H-1$, its children nodes (including $S^{\ell+1}$ ) belong to $\mathcal{L}(\Lambda) ; L^{\ell}$ is a virtual node and $T^{\ell+1}$ is a virtual leaf.

\section{Multiresolution setting}

In order to perform the multiresolution analysis, for $x \in K^{\ell}$ we define the scale function $\tilde{\varphi}_{K^{\ell}}(x):=\left|K^{\ell}\right|^{-1} \chi_{K^{\ell}}(x)$, where $\chi_{K^{\ell}}$ is the characteristic function of $K^{\ell}$, and then we can write the mean value of $u$ over $K^{\ell}$ as $u_{K^{\ell}}:=$ $\left(u, \tilde{\varphi}_{K^{\ell}}\right)_{L^{1}(\Omega)}$.

For the navigation between resolution levels, certain transfer operators are needed. These operators will allow us to determine a invertible transformation between finite volumes on level $\ell=H$, and the set formed by finite volumes on the coarsest level $\ell=0$ and a sequence of so-called wavelet coefficients. To switch from fine to coarser levels, a projection or coarsening operator is defined by the following expression

$$
u_{K^{\ell}}=\sum_{L_{i}^{\ell+1} \in \mathcal{M}_{K^{\ell}}} \frac{\left|L_{i}^{\ell+1}\right|}{\left|K^{\ell}\right|} u_{L_{i}^{\ell+1}}, \quad \tilde{\varphi}_{K^{\ell}}=\sum_{L_{i}^{\ell+1} \in \mathcal{M}_{K^{\ell}}} \frac{\left|L_{i}^{\ell+1}\right|}{\left|K^{\ell}\right|} \tilde{\varphi}_{L_{i}^{\ell+1}},
$$

where $\mathcal{M}_{K^{\ell}}$ stands for the refinement set of the control volume $K^{\ell}$, whose four elements are control volumes contained in the spatial position occupied by $K^{\ell}$, that belong to $\mathcal{T}^{\ell+1}$. To move from coarse to finer levels, a prediction operator is needed, that, as in [12], we choose to be the following polynomial reconstruction:

$$
\tilde{u}_{K^{\ell+1}}=\sum_{T^{\ell} \in \bar{S}_{K}^{\ell}} g_{K, T}^{\ell} u_{T^{\ell}}
$$

The set $\bar{S}_{K}^{\ell}$ is the stencil of interpolation (of order $s$ ) or coarsening set, $g_{K, T}^{\ell}$ are prediction coefficients, and the tilde over $u$ on the left-hand side denotes a predicted value. Details on the precise definition of these coefficients and stencils are given in e.g. [11]. For $x \in K^{\ell+1}$, and depending on the choice of the predictor map, the wavelet function is defined as

$$
\tilde{\psi}_{K^{\ell}, j}=\tilde{\varphi}_{L_{i}^{\ell+1}}-\sum_{m=-s}^{s} \tilde{\gamma}_{i+m} \tilde{\varphi}_{L_{i+m}^{l}} \quad \text { for } j=1, \ldots, 4
$$

where $L_{i}^{\ell+1} \in \mathcal{M}_{K^{\ell}}$, and the value of each $\tilde{\gamma}_{i+m}$ depends on the coefficients $g_{K, T}^{\ell}$ of the prediction operator. The error induced by the prediction operator at the finite volume $K^{\ell}$ is defined as the difference between the cell average and the predicted value, i.e., $d_{K^{\ell}}^{u}:=u_{K^{\ell}}-\tilde{u}_{K^{\ell}}$, and using the definition of the wavelet function, it holds that $d_{K^{\ell}}^{u}=\left(u, \tilde{\psi}_{K^{\ell}}\right)$. 
It is known (see e.g. [37]) that wavelet coefficients are related to the regularity of the solution, that is, if $u$ is sufficiently smooth, then its wavelet coefficients decrease when going from coarser to finer levels, so the compression of data by discarding the information corresponding to small wavelet coefficients, or thresholding procedure, is automatically achieved. In practice, the algorithm consists in discarding all control volumes corresponding to wavelet coefficients that are smaller in absolute value than a level-dependent tolerance $\varepsilon_{\ell}$,

$$
\left|d_{K^{\ell}}^{u}\right|<\varepsilon_{\ell}, \quad \ell=0, \ldots, H .
$$

Given a reference tolerance $\varepsilon_{R}$, whose choice depends on the maximum values of the reaction and diffusion coefficients (see e.g. [5] for further details on its deduction) we can determine $\varepsilon_{\ell}$ by

$$
\varepsilon_{\ell}=2^{2(\ell-H)} \varepsilon_{\mathrm{R}}, \quad \ell=0, \ldots, H .
$$

These level-dependent tolerances guarantee that the error due to thresholding is of the same order as the discretization error, and therefore the order of the underlying scheme is preserved. For multi-species problems, wavelet coefficients will be defined by $\boldsymbol{d}_{K^{\ell}}=\min \left\{\left|d_{K^{\ell}}^{u}\right|,\left|d_{K^{\ell}}^{v}\right|\right\}$ and $\boldsymbol{d}_{K^{\ell}}=\max \left\{\left|d_{K^{\ell}}^{u}\right|,\left|d_{K^{\ell}}^{v}\right|\right\}$, for the refinement and coarsening procedures, respectively.

\section{Dynamic tree structure}

A core ingredient in the fully adaptive multiresolution framework is the graded tree data storage and an efficient procedure to navigate inside it (see Figure 3). The root is the basis of the tree. A parent node has four children, and the children of the same parent are called brothers. A node without children is a leaf. A given node has $s^{\prime}=2$ nearest neighbors in each spatial direction, needed for the computation of the fluxes of leaves; if these neighbors do not exist, we create them as virtual leaves. Brothers are also considered nearest neighbors. We denote by $\Lambda$ the set of all nodes of the tree, by $\mathcal{L}(\Lambda)$ the restriction of $\Lambda$ to the leaves, and by $\mathcal{L}\left(\Lambda^{\ell}\right)$ the restriction of $\mathcal{L}(\Lambda)$ to the level $\ell$, for $\ell=0, \ldots, H$. Once the thresholding is performed, we add to the tree a safety zone, generated by adding one finer level to the tree in all leaves without violating the graded tree data structure. This is a crucial point, since it will avoid to perform the decoding of the solution back to the finest resolution level at each time step. In addition, to enforce conservativity of the scheme, we compute only the fluxes at level $\ell+1$ and we set the ingoing flux on the leaf at level $l$ equal to the sum of the outgoing fluxes on the leaves of level $\ell+1$ sharing the same edge.

Notice that in the adaptive scheme, the marching formula $(3.5)$ is performed not for all $K^{\ell} \in \Lambda^{\ell}$ but for each $K^{\ell} \in \mathcal{L}\left(\Lambda^{\ell}\right), \ell=0, \ldots, H$. We finally stress that at a given time $t=t^{n}$, by construction, the resulting adaptive mesh will be in general non-admissible. However this fact does not cause a problem. Thanks to the generation of virtual leaves, the approximate fluxes are always computed between control volumes lying on the same resolution level.

\section{Data compression rate and speed-up}

The quantity $\eta:=\mathcal{N} /\left(2^{-2 H} \mathcal{N}+\# \mathcal{L}(\Lambda)\right)$, called data compression rate [12], is used to measure the improvement in data compression. Here, $\mathcal{N}$ is the number of control volumes in the full finest grid at level $\ell=H$, and $\# \mathcal{L}(\Lambda)$ is the number of leaves in the tree. The speed-up $\mathcal{V}$ between the CPU times of the numerical solutions obtained by the one-level finite volume method and the fully adaptive multiresolution method is defined by $\mathcal{V}:=$ CPU time $\mathrm{FV} / \mathrm{CPU}$ time $_{\mathrm{MR}}$.

\begin{tabular}{cccccc}
\hline A-dimensional time & $\mathcal{V}$ & $\eta$ & $L^{1}-$ error & $L^{\infty}$-error & $L^{2}-$ error \\
\hline$\Delta t$ & & 87.02 & $7.23 \mathrm{e}^{-4}$ & $9.47 \mathrm{e}^{-4}$ & $7.52 \mathrm{e}^{-4}$ \\
10 & 11.99 & 16.88 & $8.64 \mathrm{e}^{-4}$ & $9.95 \mathrm{e}^{-4}$ & $8.43 \mathrm{e}^{-4}$ \\
100 & 16.71 & 19.48 & $8.91 \mathrm{e}^{-4}$ & $1.03 \mathrm{e}^{-3}$ & $7.65 \mathrm{e}^{-4}$ \\
800 & 18.28 & 13.21 & $9.12 \mathrm{e}^{-4}$ & $1.96 \mathrm{e}^{-3}$ & $9.83 \mathrm{e}^{-4}$ \\
1500 & 22.03 & 12.89 & $1.09 \mathrm{e}^{-3}$ & $2.36 \mathrm{e}^{-3}$ & $1.12 \mathrm{e}^{-3}$ \\
\hline
\end{tabular}

Table 1: Corresponding simulated time, CPU ratio $\mathcal{V}$, compression rate $\eta$ and normalized errors for species $u$, using the fully adaptive multiresolution method (Example 1A). 

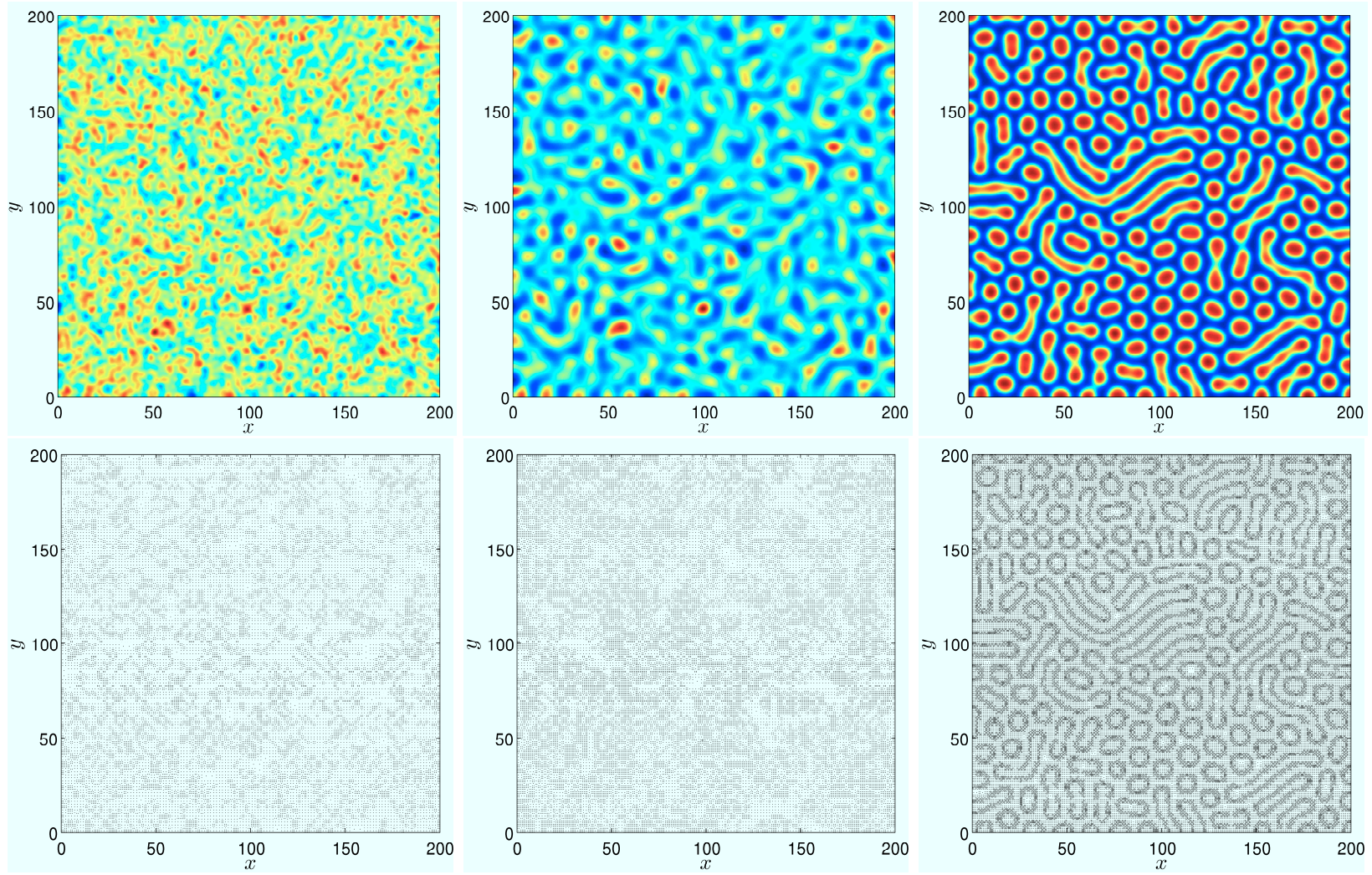

Figure 4: Numerical solution for the susceptible species $u$ (top) and leaves of the corresponding tree data structure (bottom) at time instants $t=10$, $t=100$ and $t=1500$ (Example 1A).

\section{Numerical examples}

In Example 1, Model 1 is simulated, where the parameters are chosen according to [45]. The simulation is performed using a Cartesian mesh of $\mathcal{N}=262,144$ control volumes in the highest resolution level $H=9$ and the time stepping is explicit $(\theta=0)$ with fixed time step $\Delta t=0.01$. The model parameters are set to $K=1000, \beta=0.5$, and the constant self- and cross-diffusion coefficients are chosen to be $a_{0}=0.1, b_{0}=2, c_{0}=0.02$. The reference tolerance for the multiresolution algorithm is $\varepsilon_{\mathrm{R}}=0.001$. As initial data we assume that the density of both species is a random perturbation around the endemic stationary state $\left(u^{*}, v^{*}\right)$. That is,

$$
u(x, 0)=u^{*}+u(x)_{\delta}, v(x, 0)=v^{*}+v(x)_{\delta}, \quad x \in \Omega,
$$

where $w(x)_{\delta} \in[0,1]$ is a normally distributed variable, $w \in\{u, v\}$. We will run four examples (1A, 1B, 1C and 1D) . For Examples 1A, 1B, $1 \mathrm{C}$ we set $d=0.25, r=0.27$, which gives $\left(u^{*}, v^{*}\right)=(74.0741,74.0741)$. The computational domain for Examples 1A, 1C and 1D is the square $\Omega=(0,200)^{2}$, while for Example 1B, the size of the domain has been reduced (therefore decreasing the corresponding wavenumber) to observe in more detail the behavior of the solution.

For Example 1C, the only difference to Example 1A, is that the initial data is now randomly distributed at only one spatial point, that is, we have

$$
u(x, 0)=u^{*}+u(\hat{x})_{\delta}, v(x, 0)=v^{*}+v(\hat{x})_{\delta}, \quad x \in \Omega,
$$

where $\hat{x}=(100,100)$.

We consider a fourth scenario (Example 1D) corresponding to a different set of model parameters that will also fall into a Turing space, giving raise to different spatial patterns. Here we take $d=0.15, r=0.4$, and the rest of the parameters as in Example 1A. In this case we obtain $\left(u^{*}, v^{*}\right)=(125,291.666)$. 

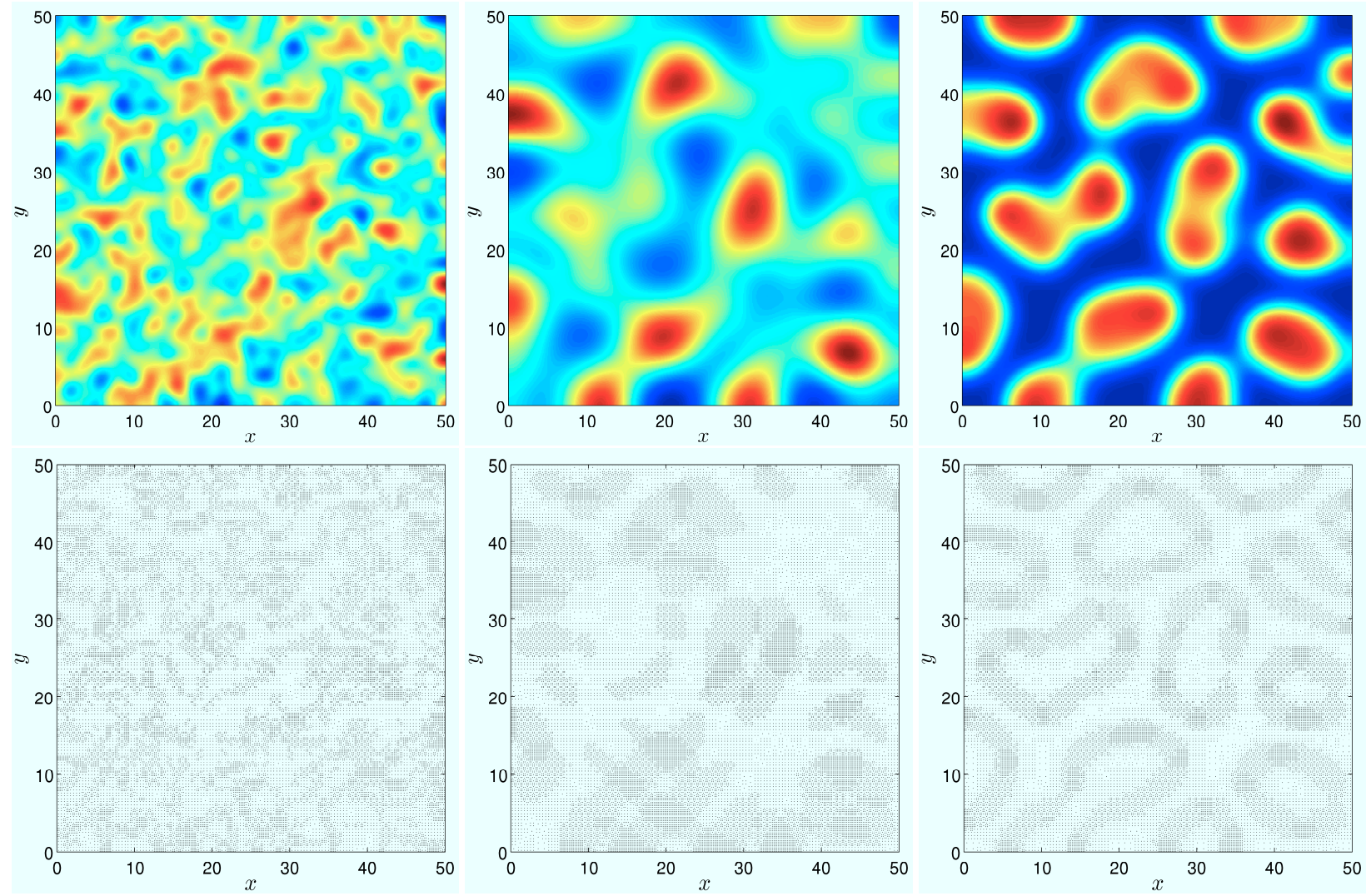

Figure 5: Numerical solution for $u$ (top) and leaves of the corresponding tree data structure (bottom) at time instants $t=5, t=125$ and $t=250$ (Example 1B).
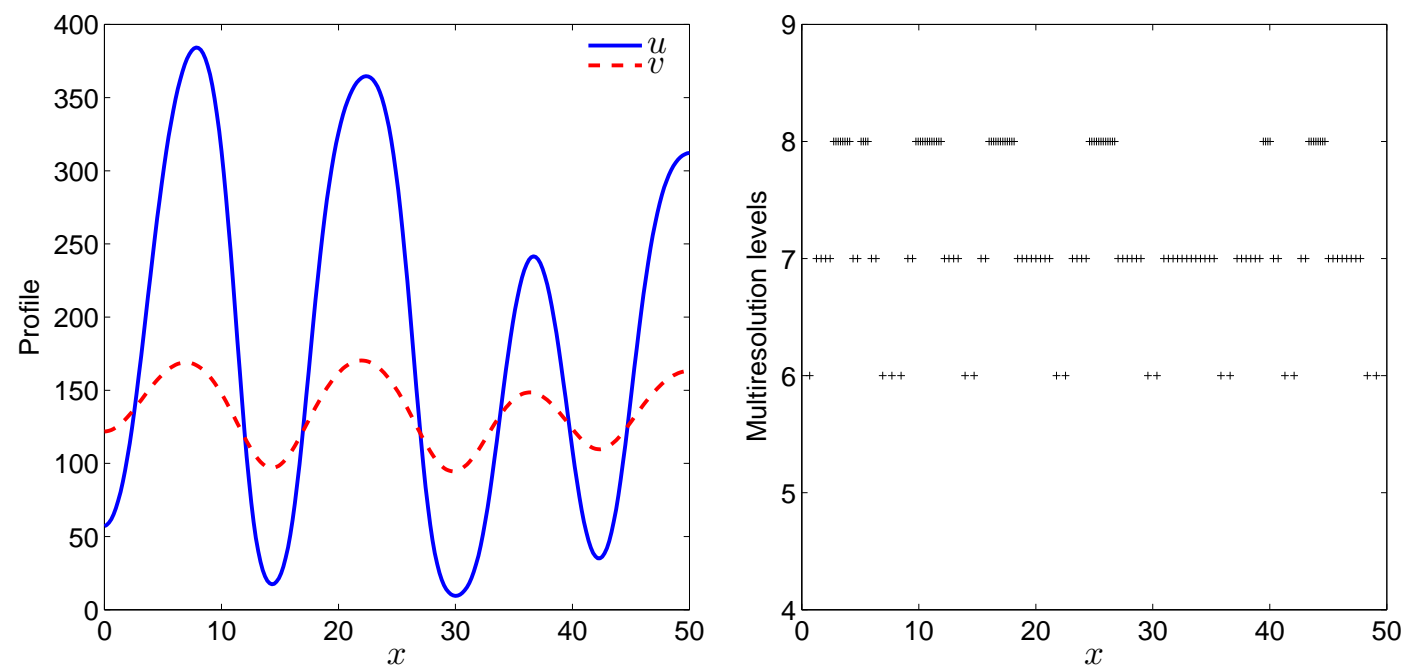

Figure 6: Profile of the numerical solution for $u$ and $v$ at $y=32.5$ (left), and leaves of the corresponding tree data structure (right) at time instant $t=250$ (Example 1B).

In Figures 4,5778 we depict snapshots of the numerical solution $u$ (the patterns of the species $v$ coincide with 

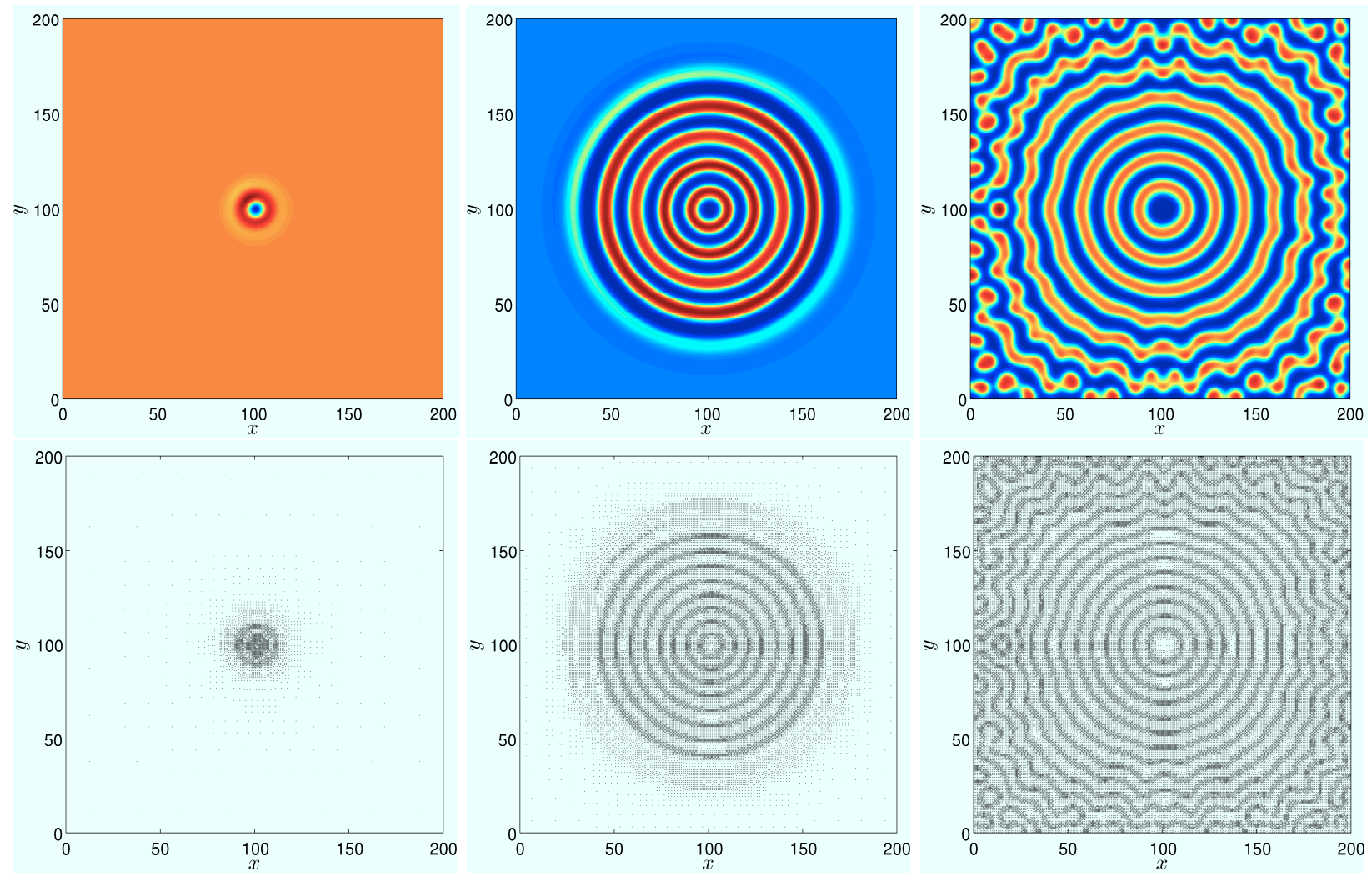

Figure 7: Numerical solution for $u$ (top) and leaves of the corresponding tree data structure (bottom) at time instants $t=100, t=400$ and $t=12000$ (Example 1C).

those of $u$, therefore it is not shown), and corresponding graded tree representation for three different time instants for Examples 1A, 1B, 1C and 1D. It can be seen how the initial random distribution of the densities evolves to reach a non-constant stationary state, that is, we observe the formation of spatial patterns induced by the Turing phenomenon. This pattern formation is remarkable since the data of Example 1 give the Jacobian matrix (2.3)

$$
F\left(u^{*}, v^{*}\right)=\left(\begin{array}{cc}
0.105 & -0.125 \\
0.125 & -0.125
\end{array}\right)
$$

which has the eigenvalues $\lambda_{1,2}=-0.01 \pm \sqrt{0.0024}$. That means that without the cross-diffusion term the solution would converge to a constant stationary state all over the two-dimensional domain.

For Examples 1A and 1B, "islands" of high concentration of susceptible individuals are formed. This reflects the phase separation triggered by the susceptible species avoiding the infected species.

In Example 1C, we notice that the perturbation in one single point leads to pattern formation in the whole domain. Furthermore, in contrast with the rest of the simulations, here the spatial patterns become clearly visible at earlier time steps. By the deterministic perturbation of the constant initial data the numerical solution becomes reproducible. In Figure 7 one can observe pattern reflection at the zero-flux but non-absorbing boundaries, that destroys the concentric structure. In order to produce solutions with more structured spatial patterns, a perturbation in two or three points would be sufficient.

As usual, the multiresolution procedure allows us to reduce the computational cost of the simulations by decreasing drastically the number of unknowns in each time step. The bottom rows in Figures $4 / 577 / 8$ give evidence of the high compression achieved by refining the mesh in zones of high gradients of the approximate solution. Further insights on the qualitative behavior of the solution (and numerical method) can be obtained by analyzing a one-dimensional analogue of Example 1B. This is displayed in Figure 6 

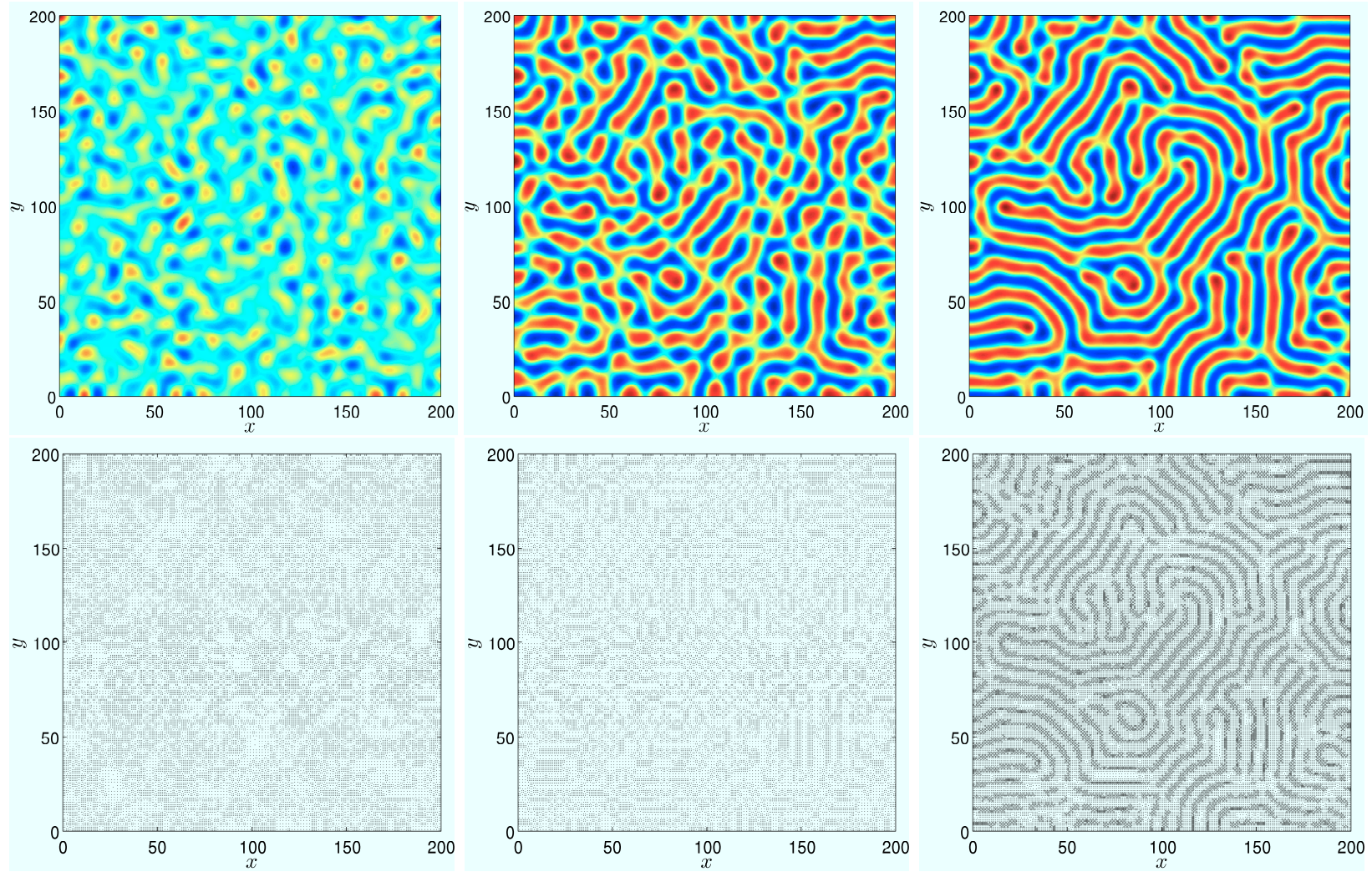

Figure 8: Numerical solution for $u$ (top) and leaves of the corresponding tree data structure (bottom) at time instants $t=100, t=800$ and $t=1500$ (Example 1D).

Table 1 illustrates the efficiency and accuracy of the adaptive method in terms of CPU ratio $\mathcal{V}$, the compression rate $\eta$, and estimated normalized errors for Example 1A. These errors correspond to the difference between the multiresolution solution reconstructed on the finest level at the final time and the uniform scheme solution on the same grid. By using the proposed multiresolution algorithm, we obtain an average data compression rate around 17, and an increasing speed-up rate up to 22. Moreover, the errors in three different norms remain of the order of $\varepsilon_{\mathrm{R}}$. Here we have computed normalized errors using a reference solution computed on a uniform mesh of 1,048,576 control volumes. To ensure that the error analysis is not affected by spurious numerical artifacts, the system has been evolved until the "random noise" which is imposed as an initial condition on the finest grid has been smoothed sufficiently and has adopted a wave-like structure; then, the solution is projected on coarser levels to obtain auxiliary initial conditions for all levels needed in order to perform the computation of errors.

In Example 2, Model 2 is simulated, where the parameters for the reaction equation are the same as in Example 1A-C. Tthe parameter of the nonlinear self-diffusion is $m=-1 / 2$. In Example 2A, the self-diffusion coefficients are set to $a_{0}=1, b_{0}=6$. For the cross-diffusion we put $c_{0}=12$, and the parameter $c_{1}$ is set to $c_{1}=u^{*}+v^{*}$ (total population in the endemic stationary state). The initial condition is now

$$
u(x, 0)=u^{*}+\rho u_{\delta}, v(x, 0)=v^{*}+\rho v_{\delta} \quad x \in \Omega,
$$

where $\rho=1 \mathrm{e}^{-4}$. The result of a qualitative comparison between Schemes A and B for Model 2 is given in Figure 10. We have computed a numerical solution of a one-dimensional problem using both schemes with a maximal resolution of 512 control volumes. Even though scheme A is based on a non-conservative discretization, one can see that the two solutions are almost indistinguishable. On the other hand, in terms of computational effort, scheme B has found to be more efficient than scheme A. Therefore, the numerical solution for the two-dimensional case has been computed using scheme B enhanced with the multiresolution strategy. In this setting, we also observe (see Figure 9) the formation of 

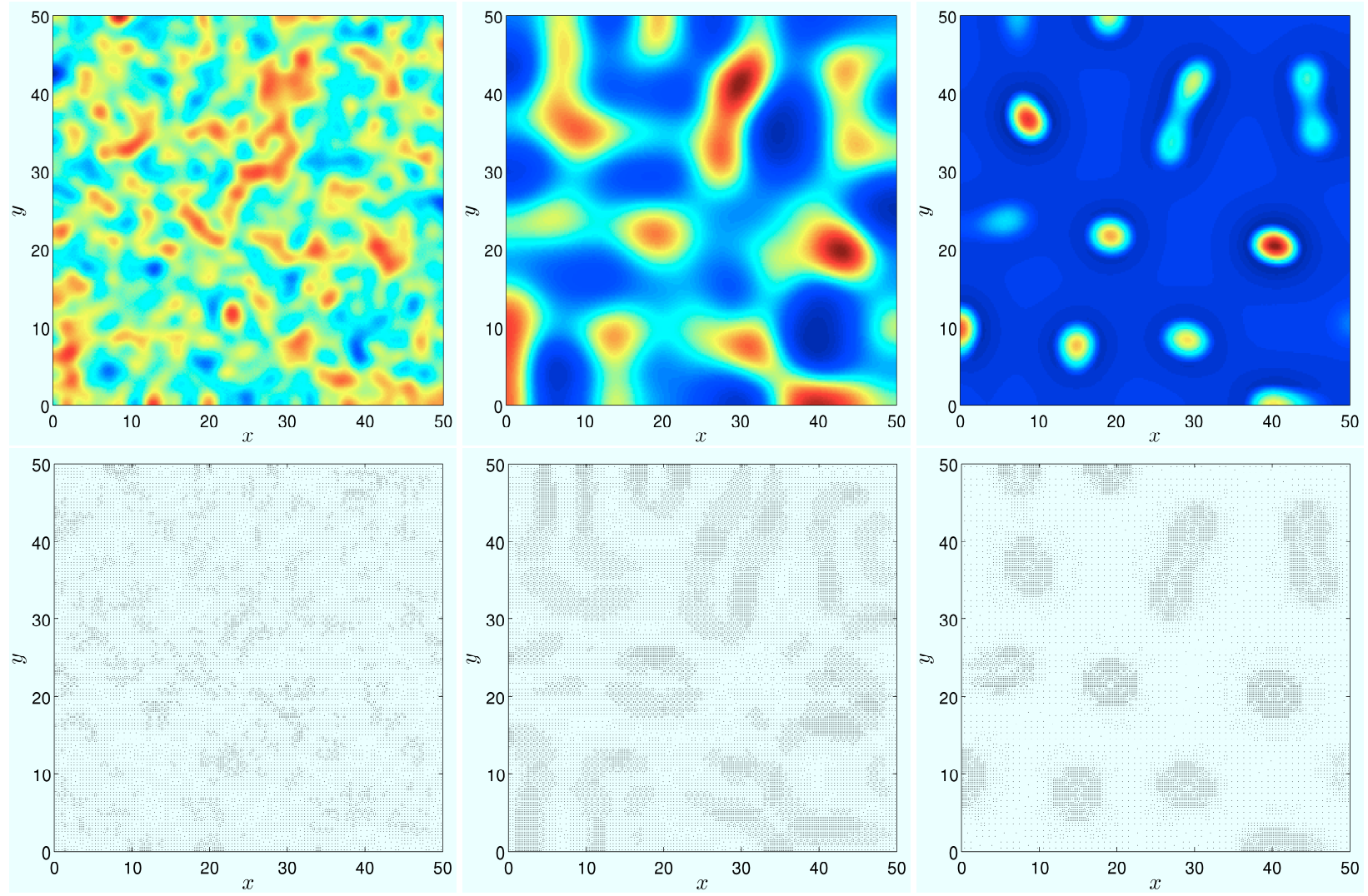

Figure 9: Numerical solution for $u$ (top) and leaves of the corresponding tree data structure (bottom) at time instants $t=0.5, t=125$ and $t=250$ (Example 2A).

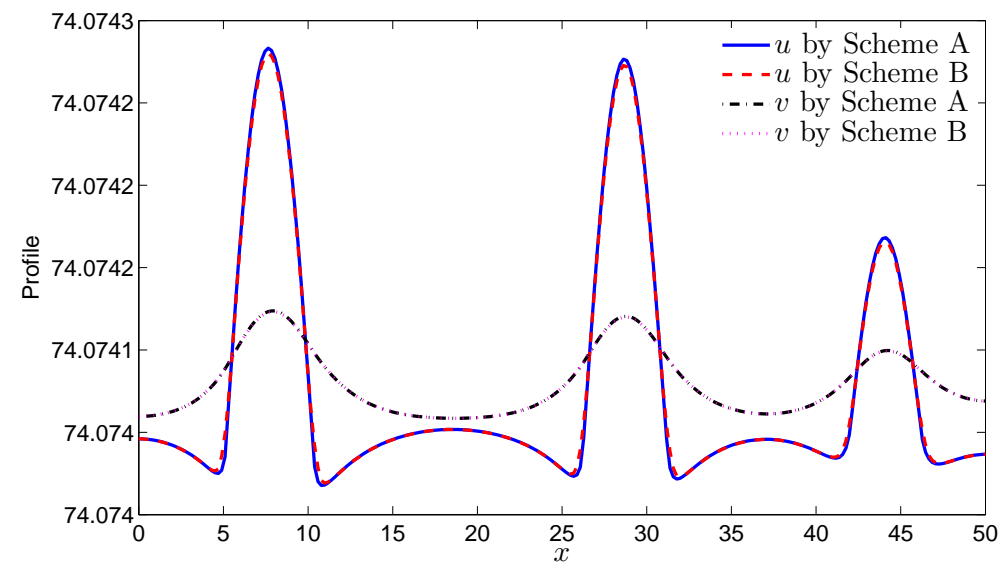

Figure 10: Profile of numerical solutions (species $u$ ) at time instant $t=250$ obtained by the one-level finite volume schemes A and B (Example 2A).

spatial patterns. Notice however, that in contrast with the results related to Model 1, here the "islands" of high concentration values of the susceptible species are surrounded by a layer of low concentration values (also noticeable from Figure 10). This behavior is in well accordance with previous contributions in the field of numerical simulation of cross-diffusion systems (see e.g. [1, 21, 22]). Finally, a study of the approximate errors is presented in Table 2

Comparing the profiles of Model 1 and Model 2 in Figures 6and 10, respectively, one recognizes that in both cases 


\begin{tabular}{cccccc}
\hline A-dimensional time & $\mathcal{V}$ & $\eta$ & $L^{1}$-error & $L^{\infty}$-error & $L^{2}$-error \\
\hline$\Delta t$ & & 39.45 & $3.91 \mathrm{e}^{-4}$ & $6.13 \mathrm{e}^{-4}$ & $3.72 \mathrm{e}^{-4}$ \\
0.5 & 17.41 & 7.45 & $4.12 \mathrm{e}^{-4}$ & $7.46 \mathrm{e}^{-4}$ & $6.55 \mathrm{e}^{-4}$ \\
5 & 19.23 & 13.62 & $4.77 \mathrm{e}^{-4}$ & $8.00 \mathrm{e}^{-4}$ & $6.80 \mathrm{e}^{-4}$ \\
125 & 26.80 & 16.35 & $6.02 \mathrm{e}^{-4}$ & $1.41 \mathrm{e}^{-3}$ & $8.15 \mathrm{e}^{-4}$ \\
250 & 31.66 & 24.11 & $6.91 \mathrm{e}^{-4}$ & $1.37 \mathrm{e}^{-3}$ & $9.06 \mathrm{e}^{-4}$ \\
\hline
\end{tabular}

Table 2: Corresponding simulated time, CPU ratio $\mathcal{V}$, compression rate $\eta$ and normalized errors for species $u$, using the fully adaptive multiresolution method combined with scheme B (Example 2A).

the maximum of species $v$ is attained as the maximum of species $u$. Whereas the profile is sinusoidal for the linear Model 1, for the nonlinear Model 2 a more refined pattern can be observed. For Model 1 the species take values in a the broad range, whereas for Model 2 the range remains close to the equilibrium. However the range can be easily manipulated by parameter calibration, given that the parametric form of the model allows a non-stationary solution, as outlined in the sequel.

To quantitatively recover the orders of magnitudes of Model 1 by the parameters for Model 2B are calibrated by the following principles.

- The state of maximum avoidance $(\hat{u}, \hat{v})$ 2.9] is close to the equilibrium $\left(u^{*}, v^{*}\right): \hat{u} \approx u^{*}, \hat{v} \approx v^{*}$.

- The coefficient $c_{0}$ is chosen such that the cross-diffusion coefficient at the equilibrium $c_{0} u^{*} v^{*}\left(c_{1}-u^{*}-v^{*}\right)$ is larger than the coefficient in the corresponding linear parametrization. The idea is that the nonlinear crossdiffusion coefficient has in average the same value as the reference linear cross-diffusion coefficient.

The latter principle can be realized as follows: In the first instance one has to assure that $c_{1} \gg u^{*}+v^{*}$ such that $c_{1}-u^{*}+v^{*} \gg 0$ as a non-vanishing value. Then one has to adjust $c_{0}$ such that $c_{0} u v\left(c_{1}-u^{*}-v^{*}\right) \approx 0.02$, i.e the model 2 reflects the values of model 1 .

In consequence, for Example 2B (see Figure 11$)$ we choose $a_{0}=0.5, b_{0}=3, c_{1}=3 u^{*}, c_{0}=0.02\left(u^{*} v^{*}\left(c_{1}-\right.\right.$ $\left.\left.u^{*}-v^{*}\right)\right)^{-1}$, and the remaining parameters as in Example 1B. From Figure 12 we notice that the solution recovers the same scaling as in Example 1A.

\section{Conclusions}

In this paper we have proposed a nonlinear cross-diffusion model for epidemic dynamics. The ordinary differential equations involving the proposed reaction terms of our epidemic model are asymptotically stable in the sense that if the initial data are chosen close to the equilibrium then the solution converges to the equilibrium. The numerical examples of the two-dimensional reaction-diffusion equation show that there is a spacial phase separation in spite of the convergence behavior of the pure reaction terms. This means that the cross-diffusion in the parabolic terms of the reaction-diffusion equation is "stronger" than the attraction of the reaction terms. This is a remarkable property in comparison with cross-diffusion models where the ordinary differential equations represented by the reaction part only show Lyapunov stability in the sense that initial data chosen in a close neighborhood of the equilibrium then the solution remains in this neighborhood.

For the simulation both a conservative and a non-conservative discretization have been proposed; both produce the same limit solution. The proposed schemes work stable both for the linear and nonlinear equations. The fully adaptive numerical method is particularly efficient to resolve phase interfaces due to the adaptive strategy.

From the numerical viewpoint, possible straightforward improvements include the use of time adaptive strategies such as local time stepping or Runge-Kutta-Fehlberg methods [5], or the use of a multiresolution analysis defined on general unstructured meshes [43].

From the model point of view one further issue is to choose different reaction kinetics in order study quantitatively how the pattern formation produced by the cross-diffusion term can be compensated by a stronger asymptotical stability of the reaction ODEs. 

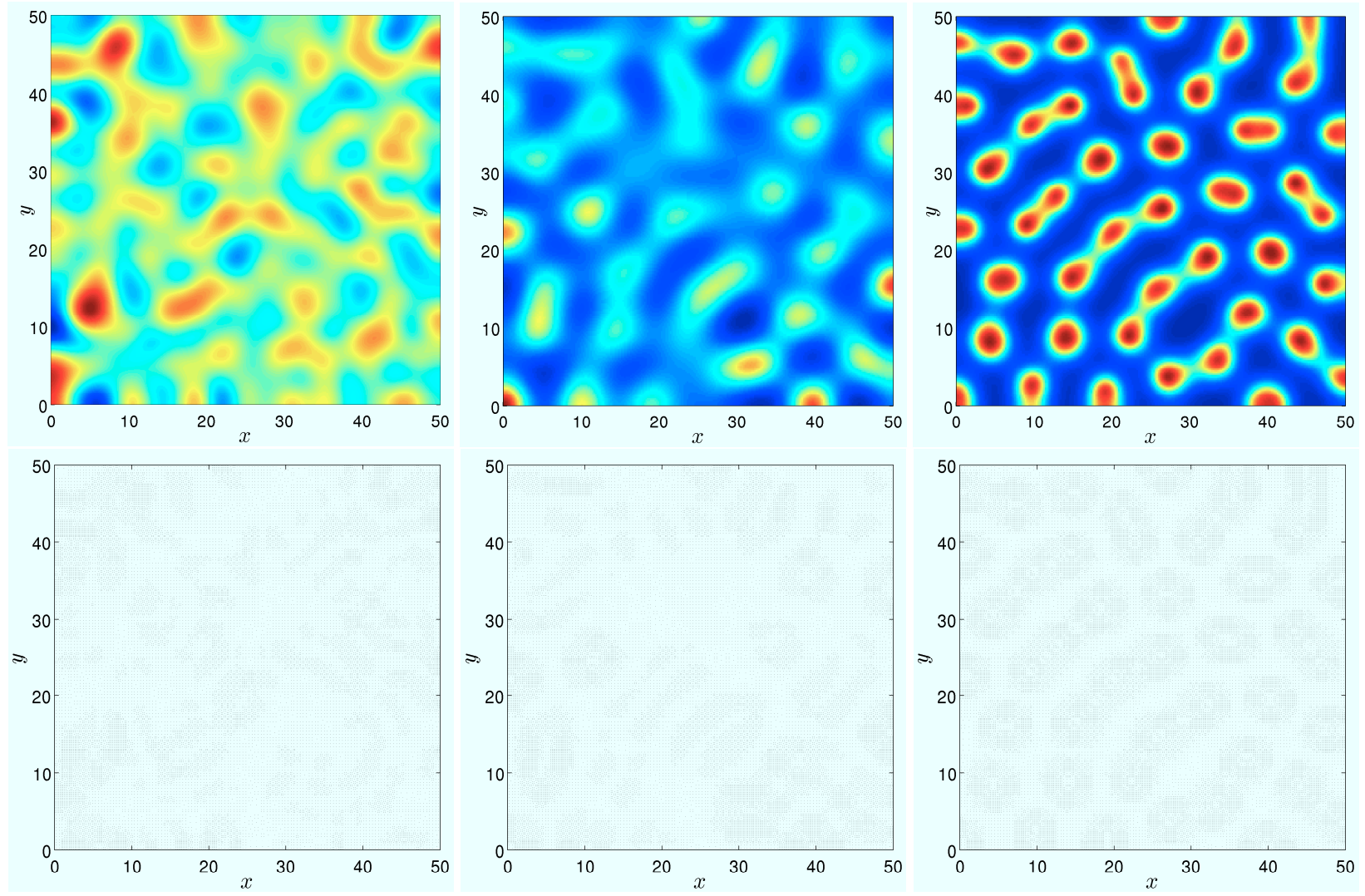

Figure 11: Numerical solution for $u$ (top) and leaves of the corresponding tree data structure (bottom) at time instants $t=50, t=250$ and $t=750$ (Example 2B).

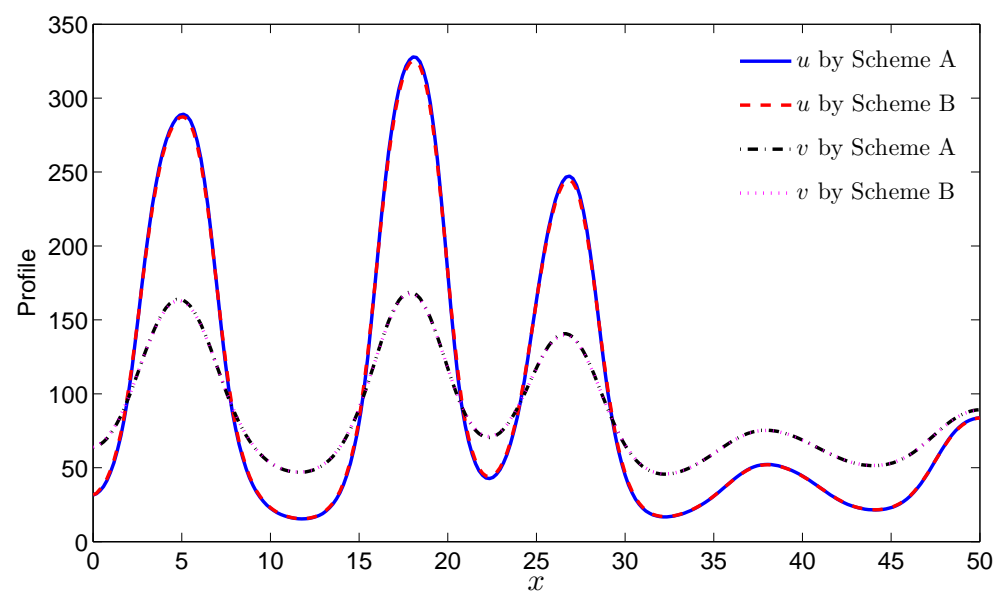

Figure 12: Profile of numerical solutions (species $u$ ) at time instant $t=750$ obtained by the one-level finite volume schemes A and B (Example 2B).

\section{Acknowledgments}

The first author is supported by Conicyt (Chile) through Fondecyt project \#11080253. The work of the second author is supported by the European Research Council Advanced Grant "Mathcard, Mathematical Modelling and 
Simulation of the Cardiovascular System", Project ERC-2008-AdG 227058.

\section{References}

[1] B. Andreianov, M. Bendahmane, R. Ruiz-Baier, Analysis of a finite volume method for a cross-diffusion model in population dynamics, Math. Meth. Models Appl. Sci., to appear.

[2] S. Badraoui, Existence of global solutions for systems of reaction-diffusion equations on unbounded domains, Electron. J. Diff. Eqn. 2002 (2002) 1-10.

[3] S. Badraoui, Asymptotic behavior of solutions to a $2 \times 2$ reaction-diffusion system with a cross diffusion matrix on unbounded domains, Electron. J. Diff. Eqn. 2006 (2006) 1-13.

[4] J.W. Barrett, J.F. Blowey, Finite element approximation of a nonlinear cross-diffusion population model, Numer. Math. 98 (2004) 195-221.

[5] M. Bendahmane, R. Bürger, R. Ruiz-Baier, K. Schneider, Adaptive multiresolution schemes with local time stepping for two-dimensional degenerate reaction-diffusion systems, Appl. Numer. Math. 59 (2009) 1668-1692.

[6] M. Bendahmane, T. Lepoutre, A. Marrocco, B. Perthame, Conservative cross diffusions and pattern formation through relaxation, J. Math. Pures Appl. 92 (2009) 651-667.

[7] M. Bendahmane, M. Sepúlveda, Convergence of a finite volume scheme for nonlocal reaction-diffusion systems modelling an epidemic disease, Disc. Cont. Dynam. Syst. - Series B 11 (2009) 823-853.

[8] S. Berres, R. Bürger, H. Frid, Neumann problems for quasi-linear parabolic systems modeling polydisperse suspensions, SIAM J. Math. Anal. 38 (2006) 557-573.

[9] R. Bürger, S. Evje, K.H. Karlsen, K.-A. Lie, Numerical methods for the simulation of the settling of flocculated suspensions, Chem. Eng. J. 80 (2000) 91-104.

[10] R. Bürger, R. Ruiz-Baier, Multiresolution simulation of reaction-diffusion systems with strong degeneracy, Bol. Soc. Esp. Mat. Apl. SëMA 47 (2009) 73-80.

[11] R. Bürger, R. Ruiz-Baier, K. Schneider, Adaptive multiresolution methods for the simulation of waves in excitable media, J. Sci. Comput., in press.

[12] R. Bürger, R. Ruiz-Baier, K. Schneider, M. Sepúlveda, Fully adaptive multiresolution schemes for strongly degenerate parabolic equations in one space dimension, M2AN Math. Model. Numer. Anal. 42 (2008) 535-563.

[13] M.J. Castro, J.M. Gallardo, C. Parés, High-order finite volume schemes based on reconstruction of states for solving hyperbolic systems with nonconservative products. Applications to shallow-water systems, Math. Comp. 75 (2006) 1103-1134.

[14] M.C. Chubb, K.H. Jacobsen, Mathematical modeling and the epidemiological research process, Eur. J. Epidem. 25 (2009) 13-19.

[15] M. Crandall, A. Pazy, L. Tartar, Global existence and boundedness in reaction-diffusion systems, SIAM J. Math. Anal. 18 (1987) 744-761.

[16] E.H. Daddiouaissa, Existence of global solutions for a system of reaction-diffusion equations having a triangular matrix, Electron. J. Diff. Eqn. 2008 (2008) art. 141.

[17] S.F. Dowell, Seasonal variation in host susceptibility and cycles of certain infectious diseases, Emerg. Infect. Dis. 7 (2001) 369-374.

[18] R. Eymard, T. Gallouët, R. Herbin, Finite Volume Methods. In: P.G. Ciarlet, J.L. Lions (eds.), Handbook of Numerical Analysis, vol. VII, North-Holland, Amsterdam (2000) 713-1020. 
[19] H. Frid, V. Shelukhin, A quasi-linear parabolic system for three-phase capillary flow in porous media, SIAM J. Math. Anal. 35 (2004) 1029-1041.

[20] H. Frid, V. Shelukhin, Initial boundary value problems for a quasi-linear parabolic system in three-phase capillary flow in porous media, SIAM J. Math. Anal. 36 (2005) 1407-1425.

[21] G. Galiano, M.L. Garzón, A. Jüngel, Semi-discretization and numerical convergence of a nonlinear crossdiffusion population model, Numer. Math. 93 (2003) 655-673.

[22] G. Gambino, M.C. Lombardo, M. Sammartino, A velocity-diffusion method for a Lotka-Volterra system with nonlinear cross and self-diffusion, Appl. Num. Math. 59 (2009) 1059-1074.

[23] A. Hastings, Can spatial variation alone lead to selection for dispersal?, Theoret. Popul. Biol. 24 (1983) $244-251$.

[24] B.T. Hayes, P.G. LeFloch, Non classical shocks and kinetic relations: Finite difference schemes, SIAM J. Numer. Anal. 35 (1998) 2169-2194.

[25] D. He, L. Stone, Spatio-temporal synchronization of recurrent epidemics, Proc. Roy. Soc. Lond. B 270 (2003) 1519-1526.

[26] H.W. Hethcote, Mathematics of infectious diseases, SIAM Rev. 42 (2000) 599-653.

[27] H.W. Hethcote, P. Van Den Driessche, Two SIS epidemiologic models with delays, J. Math. Biol. 40 (2000) 3-26.

[28] W.O. Kermack, A.G. McKendrick, A contribution to the mathematical theory of epidemics, Proc. Roy. Soc. Lond. A 115 (1927) 700-721.

[29] K.I. Kim, Z. Lin, L. Zhang, Avian-human influenza epidemic model with diffusion, Nonl. Anal.: Real World Appl. 11 (2010) 313-322.

[30] P.D. Lax, B. Wendroff, Systems of conservation laws, Comm. Pure Appl. Math. 13 (1960) 217-237.

[31] J. Li, X. Zou, Modeling spatial spread of infectious diseases with a fixed latent period in a spatially continuous domain, Bull. Math. Biol. 71 (2009) 2048-2079.

[32] K. Li, M. Small, H. Zhang, X. Fu, Epidemic outbreaks on networks with effective contacts, Nonl. Anal.: Real World Appl. 11 (2010) 1017-1025.

[33] J. Liu, T. Zhang, Analysis of a nonautonomous epidemic model with density dependent birth rate, Appl. Math. Model. 34 (2010) 866-877.

[34] A.L. Lloyd, R.M May, How viruses spread among computers and people, Science 292 (2001) 1316-1317.

[35] L. Melkemi, A.Z. Mokrane, A. Youkana, On the uniform boundedness of the solutions of systems of reactiondiffusion equations, Electron. J. Qual. Th. Diff. Eqn. 2005 (2005) 1-10.

[36] F.A. Milner, R. Zhao, S-I-R model with directed spatial diffusion, Math. Popul. Stud. 15 (2008) 160-181.

[37] S. Müller, Adaptive Multiscale Schemes for Conservation Laws, Springer-Verlag, Berlin (2003).

[38] W.-M. Ni, Diffusion and cross-diffusion in pattern formation, Rend. Mat. Acc. Lincei 15 (2004) $197-214$.

[39] M. Nuño, C. Castillo-Chavez, Z. Feng, M. Martcheva, Mathematical models of influenza: The role of crossimmunity, quarantine and age-structure, in: Lecture Notes in Mathematics 1945 (2008) 349-361.

[40] R.S. Ostfeld, G.E. Glass, F. Keesing, Spatial epidemiology: An emerging (or re-emerging) discipline, Trends Ecol. Evol. 20 (2005) 328-336.

[41] C. Parés, Numerical methods for nonconservative hyperbolic systems: A theoretical framework, SIAM J. Numer. Anal. 44 (2006) 300-321. 
[42] S. Phongthanapanich, P. Dechaumphai, Finite volume element method for analysis of unsteady reaction-diffusion problems, Acta Mech. Sin. 25 (2009) 481-489.

[43] F. Schröder-Pander, T. Sonar, and O. Friedrich, Generalized multiresolution analysis on unstructured grids, Numer. Math. 86 (2000) 685-715.

[44] L.W. Somathilake, J. Peiris, Global solutions of a strongly coupled reaction-diffusion system with different diffusion coefficients, J. Appl. Math. 1 (2005) 23-36.

[45] G.-Q. Sun, Z. Jin, Q.-X. Liu, L. Li, Spatial pattern in an epidemic system with cross-diffusion of the susceptible, J. Biol. Syst. 17 (2009) 141-152.

[46] C. Tian, Zh. Lin, M. Pedersen, Instability induced by cross-diffusion in reaction-diffusion systems, Nonl. Anal.: Real World Appl. 11 (2010) 1036-1045.

[47] W. Wang, S. Ruan, Bifurcations in an epidemic model with constant removal rate of the infectives, J. Math. Anal. Appl. 291 (2004) 775-793.

[48] G. Webb, A reaction-diffusion model for a deterministic diffusive epidemic, J. Math. Anal. Appl. 84 (1981) 150-161.

[49] J.C. Wong, The Galerkin finite element method for the solution of some spatio-temporally dependent reactiondiffusion systems, Appl. Numer. Math. 58 (2008) 352-375. 